\title{
Numerical solution of photocounting statistics inverse problem for few-photon pulsed laser sources
}

\author{
Pavel Gostev ${ }^{1, *}$, Sergey Magnitskiy ${ }^{1}$, Anatoly Chirkin ${ }^{1}$, and Andrey Demin ${ }^{2}$ \\ ${ }^{1}$ Lomonosov Moscow State University, Department of Physics and International Laser Center, 119991 \\ Moscow, Russia \\ ${ }^{2}$ The All-Russian Research Institute for Optical and Physical Measurements, 119361 Moscow, Russia
}

\begin{abstract}
We present a numerical procedure for recovering of photon statistics of few-photon pulsed laser sources. It provides controlled level of mean error for both of photon and photocounting statistics. It is shown that procedure is correct for model data with average error level of $10^{-4}$ and fidelity 0.9998 .
\end{abstract}

The task of characterizing pulsed few-photon laser sources is now relevant for many areas of quantum technology: quantum cryptography [1], production of nanolasers [2], etc. Such devices are necessary both for fundamental science and for the development of technology, which means that they should be strictly characterized. In this work, we pay attention to the technique of measuring the energy characteristics of few-photon pulsed laser sources through the statistics of photocounts obtained by MPPC [3]. We propose an algorithm that allows us to estimate the energy spectrum - the photon-number statistics of the source under study.

As is known, the statistics of the number of photons in the pulses of an ideal pulsed laser $P_{\text {sig }}$ obeys the Poisson statistics [4]. Due to internal noise, instability of parameters and spontaneous luminescence of the active medium, statistics may be distorted. It is reasonable to adopt a model of independent additive noise, in which the distribution of light $P(n)$ with fixed mean $\bar{n}$ is described by the convolution of the partial distributions of the signal $P_{\text {sig }}$ and noise $P_{n s e}$ :

$$
P=P_{\text {sig }} * P_{n s e}
$$

In the case of finite quantum efficiency $\eta$, the photon statistics in the general case differs from the statistics of photocounts $q$, however, it is connected with it as [5]:

$$
\begin{aligned}
& q(m)=T_{m n} P(n) \\
& P(n)=T_{m n}^{-1} q(m)
\end{aligned}
$$

where $T_{m n}=C_{n}^{m} \eta^{n}(1-\eta)^{n-m}$ is an upper triangular matrix. However, the task of restoring $P(n)$ according to formula (2b) is ill-posed [6], that leads to uncontrolled errors when it is used to restore $P(n)$ from experimentally measured values of $q(m)$. Because of that we suggest finding an estimation to $P$ iteratively via optimizing a direct problem (2a) in a bilevel

\footnotetext{
*Corresponding author: gostev.pavel@physics.msu.ru
} 
approach. $P$ can be described as a variable with length $\mathrm{N}$ that maximizes the fidelity:

$$
F(P)=\sum_{m} \sqrt{T_{m n} P(n)} \sqrt{q(m)}
$$

Here photocounting probability $q(m)$ has length $\mathrm{M}$; matrix T has dimensions (N, M). The lower-level optimization problem can be described as follows:

$$
\left\{\begin{array}{l}
\text { maximize: } F(P) \\
\text { subject to: } b_{l} \leq P \leq b_{u}, \\
\|P\|=1,\left\|T_{m n} P\right\|=1, \\
\quad\left\langle m^{(r)} \mid q\right\rangle=\eta^{r}\left\langle n^{(r)} \mid P\right\rangle, r=1 \ldots N_{r}
\end{array}\right.
$$

We assume that excess noise is thermal with mean $\bar{n}_{t h}$. Because of uncertainty of measurement we know $\bar{n}_{t h}$ with finite precision, so we must set lower $\bar{n}_{t h}^{(l)}$ and upper $\bar{n}_{t h}^{(u)}$ bounds for $\bar{n}_{t h}$. Then bounds $b_{l}, b_{u}$ can be determined as $b_{l}=\min \left(p_{l}, p_{u}\right), b_{u}=$ $\max \left(p_{l}, p_{u}\right)$, where $p_{l}=P_{\text {sig }}\left[\bar{n}-\bar{n}_{t h}^{(l)}\right] * P_{n s e}\left[\bar{n}_{t h}^{(l)}\right], p_{u}=P_{\text {sig }}\left[\bar{n}-\bar{n}_{t h}^{(u)}\right] * P_{n s e}\left[\bar{n}_{t h}^{(u)}\right]$.

Hence the problem (4) has a conditional fitness function $F\left(P \mid \bar{n}_{t h}^{(l)}, \bar{n}_{t h}^{(u)}\right)$ and its optimal value depends from $\bar{n}_{t h}^{(l)}$ and $\bar{n}_{t h}^{(u)}: \max _{P} F\left(P \mid \bar{n}_{t h}^{(l)}, \bar{n}_{t h}^{(u)}\right)=V\left(\bar{n}_{t h}^{(l)}, \bar{n}_{t h}^{(u)}\right)$. Therefore, we can write the upper-level optimization problem:

$$
\left\{\begin{array}{l}
\text { maximize: } V\left(\bar{n}_{t h}^{(l)}, \bar{n}_{t h}^{(u)}\right) \\
\text { subject to: } \bar{n}_{t h}^{(u)} \in[0, \bar{n}], \bar{n}_{t h}^{(l)} \in\left[0, \bar{n}_{t h}^{(u)}\right]
\end{array}\right.
$$

The problem (5) is optimized with particle swarm method [7].

The algorithm was implemented in Python 3 using the pyomo optimization framework [8] and the software library for nonlinear optimization IPOPT [9] with the HSL MA97 solver [10]. To check the correctness of the algorithm, we used model statistics of photons - a convolution of the Poisson $(\langle n\rangle=5)$ and thermal $(\langle n\rangle=1)$ distributions. To simulate the experimental situation when solving the inverse problem, all the values of $P^{(e)}(m \geq 10)$ which were obtained in the direct calculation were cut off, and additionally the noise with amplitude of $5 \times 10^{-5}$ and with zero mean was added to $P^{(e)}(m)$. For comparison, the same calculation was performed according to formula (2b). The average error for the proposed algorithm was turned to be $10^{-4}$ with fidelity $F=0.9998$, while for the analytical formula (2b) an error of order of 66.6 or approximately $3 \cdot 10^{4} \%$ was obtained.

This work was partially supported by the RFBR grant 18-02-00849.

\section{References}

1. C. J. Chunnilall, Standard: ETSI - GS QKD 011 (ETSI, 2016)

2. E. Wei, Senior Thesis in Electrical Engineering (University of Illinois at UrbanaChampaign, 2013)

3. S. Magnitskiy, et al., Metrology of photon statistics of pulsed low-photon light sources (2018 International Conference Laser Optics, IEEE, 2018)

4. R. S. Bondurant, et al., Opt. letters, 7, 11 (1982)

5. M. O. Scully, E. Willis, J. R. Lamb, Phys. Rev. V, 179, 2 (1969)

6. D. N. Klyshko, M. Chekhova, S. Kulik, Physical foundations of quantum electronics (World Scientific, 2011)

7. J. Kennedy, Particle swarm optimization (Encyclopedia of machine learning, 2010)

8. K. Kaminishi, et al., Phys. Rev. A, 24, 1 (1981)

9. F. E. Curtis, et al., Math. Prog., 136, 1 (2012)

10. J. D. Hogg, J. A. Scott, HSL_MA97 (Science and Technology Facilities Council, 2011) 